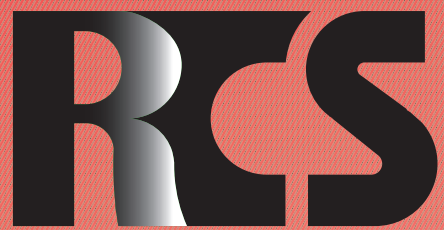

Depósito legal ppi $201502 Z U 4662$

Esta publicación científica en formato digital es continuidad de la revista impresa Depósito Legal: pp $197402 Z U 789$

- ISSN: 1315-9518 • ISSN-E: 2477-9431

Revista de Ciencias Sociales

Universidad del Zulia. Revista de la Facultad de Ciencias Económicas y Sociales Vol. XXVII. No. 2

Abril-Junio 2021

Esta publicación científica en formato digital es continuidad de la revista impresa Depósito Legal: pp $197402 Z$ Z789 ISSN: 1315-9518 


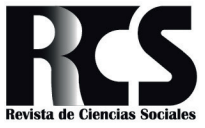

Revista de Ciencias Sociales (RCS). FCES - LUZ

Vol. XXVII, No. 2, 2021, pp. 217-227 Abril-Junio

- ISSN: 1315-9518 IISSN-E: 2477-9431
Como citar APA: Parra, M., Rocha, G., y Durán, S. (2021). Liderazgo como prospectiva del clima organizacional en el sector hotelero. Revista de Ciencias Sociales (Ve), XXVII(2), 217-227.

\title{
Liderazgo como prospectiva del clima organizacional en el sector hotelero
}

\author{
Parra, Margel"

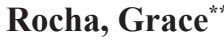 \\ Durán, Sonia ${ }^{* * *}$
}

\section{Resumen}

El estudio se orientó a analizar el liderazgo como prospectiva del clima organizacional en el sector hotelero de Cartagena de Indias. Se sustentó teóricamente en Robbins y Judge (2017), Brunet (2011), entre otros. El tipo de investigación fue descriptiva de campo, con un diseño no experimental, transversal. La población estuvo constituida por una muestra de 35 empleados operativos del sector hotelero de Cartagena, a quienes se les aplicó un cuestionario con escala Likert. El instrumento fue validado según el juicio de 5 expertos y la confiabilidad se destaca según el método de Crombach, cuyo resultado es 0,84 . El análisis de datos se realizó mediante estadísticas descriptivas, utilizando un baremo de interpretación, construido bajo las bases de puntajes mínimos y máximos de la escala utilizada. Se presentan las tendencias por variable, posterior la correlación entre ella. Como resultado se concibe que, en el sector hotelero el estilo de liderazgo genera una relación con el clima organizacional predominante con un coeficiente de Pearson de 0,86 , determinando una relación muy significativa. Se concluyó que, en las organizaciones objeto de estudio el estilo de liderazgo es generalmente autoritario y cerrado, lo que implica tensiones en el ambiente y en la ejecución de los procesos.

Palabras clave: Ambiente; clima organizacional; desempeño; liderazgo; sector hotelero.

\footnotetext{
* Candidata a Doctora en Ciencias Sociales. Docente de la Corporación Universitaria Antonio José de Sucre, adscrita al Grupo de Investigación GINCIS, Universidad de la Costa, Colombia. E-mail: docente investigador11@corposucre. edu.co iD ORCID: https://orcid.org/0000-0003-3530-8112

** Magister en Desarrollo Organizacional y Procesos Humanos. Docente de la Universidad de la Costa, Colombia. E-mail: grocha@cuc.edu.co iD ORCID: https://orcid.org/0000-0002-2635-5067

*** Doctora en Ciencias Gerenciales. Docente Investigador en la Facultad de Derecho y Ciencias de la Universidad Libre, campus Barranquilla, Colombia, Adscrita al grupo de investigación: INCOM. Investigaciones de Derecho y Educación. E-mail: sonia.etheld@unilibre.edu.co iD ORCID: https://orcid.org/0000-0002-0876-7850
} 


\title{
Leadership as a prospect of the organizational climate in the hotel sector
}

\begin{abstract}
The study was aimed at analyzing leadership as a prospective of the organizational climate in the hotel sector of Cartagena de Indias. It was theoretically supported by Robbins and Judge (2017), Brunet (2011), among others. The type of research was descriptive in the field, with a non-experimental, crosssectional design. The population consisted of a sample of 35 operational employees of the Cartagena hotel sector, to whom a Likert scale questionnaire was applied. The instrument was validated according to the judgment of 5 experts and the reliability stands out according to the Crombach method, whose result is 0.84 . The data analysis was carried out through descriptive statistics, using an interpretation scale, built on the basis of the minimum and maximum scores of the scale used. The trends by variable are presented, after the correlation between them. As a result, it is conceived that, in the hotel sector, the leadership style generates a relationship with the predominant organizational climate with a Pearson coefficient of 0.86 , determining a very significant relationship. It was concluded that, in the organizations under study, the leadership style is generally authoritarian and closed, which implies tensions in the environment and in the execution of the processes.
\end{abstract}

Keywords: Environment; organizational climate; performance; leadership; hotel sector.

\section{Introducción}

En el trabajo decisivo de formular, implementar, revisar y actualizar estrategias, la disciplina del líder debe estar llena de pasión, habilidad y compromiso para asegurar la posibilidad de supervivencia y alcanzar el nivel de éxito organizacional. Entre estas visiones, Parra, et al. (2019) consideran que parte de la gestión organizacional incluye el fortalecimiento de las condiciones laborales y personales, con el fin de integrar a los empleados en la organización, abarcando características de los empleados como habilidades, destrezas, entusiasmo y lealtad. En grado, estos afectarán los resultados, la eficiencia, la reputación y la supervivencia de la organización.

En función de lo anteriormente expuesto, García, et al. (2017a), asumen que el talento humano en una empresa desempeña funciones para lograr las metas deseadas; por lo cual, se hace necesario contar con un ambiente de trabajo capaz de ofrecer condiciones positivas, que generen beneficios de forma particular coadyuvando al desarrollo y progreso organizacional, sustentado por Robbins y Judge (2017). Cabe destacar, que el concepto de ambiente desde la perspectiva organizacional, se relaciona con el clima organizacional, en este ámbito es apreciado por Chiavenato (2013), como la atmósfera psicológica particular ligada a la satisfacción de las necesidades humanas de sus miembros.

Con base en lo anterior, García, et al. (2017a) asumen que los talentos de la empresa pueden alcanzar las metas deseadas, para lo cual, debe existir una condición positiva que pueda brindar ambiente de trabajo. Con el apoyo de Robbins y Judge (2017), los beneficios generados de manera específica contribuyen al desarrollo y progreso organizacional. En ese sentido, el concepto de ambiente desde la perspectiva de la organización, se relaciona con el ambiente de la misma; al respecto, Chiavenato (2013) cree que se trata de un ambiente psicológico especial relacionado con la satisfacción de las necesidades humanas de sus integrantes.

Este podría relacionarse con los estilos de liderazgo, en este proceso se estimula al personal para que realice su trabajo; en ese 
sentido, Parra, et al. (2018), han considerado en su estudio de Clima organizacional, la importancia de un proceso de interacción coordinada, donde el sistema técnico, el sistema humano, y el ambiente, se conectan para desarrollar actividades, acciones así como procesos generales y que de una manera determinada afectan las particularidades en cada sujeto. En este sentido, el clima refiere y se genera desde la percepción del empleado, presentándose como una técnica mediadora entre la empresa y los trabajadores.

Martínez-Ventura, et al. (2017) consideran que las organizaciones deben brindar orientación y apoyo a los empleados para alinear sus metas con las metas generales de estos; por lo que, se debe mantener un estilo de liderazgo consistente para las necesidades de los procesos organizacionales. En consecuencia, estas empresas deben guiarse por estilos de liderazgo participativo, que transformen los procesos, tomando como uno de los pilares básicos de sus actividades empresariales al empleado, así como comprender a los líderes en sus escenarios de organización de acción, pero también entender la competitividad.

Pero paralelo a cumplir con un objetivo de servicio al cliente externo, estas organizaciones también tienen que vincularse con su personal y ofrecer ambientes laborales estables, que generen calidad y bienestar laboral, lo que circunscribe la existencia de un líder que sea agente mediador entre la empresa y los trabajadores.

De acuerdo con lo planteado, el ambiente laboral en el sector hotelero de Cartagena (Colombia) se presenta desde una perspectiva compleja, originado por la misma razón del negocio y las actividades que ejecutan. Indudablemente, que la ciudad vive en constante proceso vacacional, aunque hay unas temporadas que tienden a ser bajas, la situación de destino turístico de alto impacto, determina que en Cartagena los criterios del sector son siempre orientados a temporada alta, por lo cual, debe ofrecerse un servicio de la más alta calidad a miles de turistas nacionales e internacionales, respondiendo de manera exitosa al mercado meta.

Ahora bien, se ha evidenciado en el sector una serie de dificultades, que han afectado el rol de los líderes, quienes han ido generando imposición de procesos, manifiestos en la debilidad para formular, desarrollar, hacer seguimiento y control de la gestión del servicio, estableciendo una excesiva centralización de las decisiones, mediante las cuales se restringe la capacidad de iniciativa y de acción del personal tanto supervisorio, como operativo.

Se ha revelado que los empleados generalmente están sometidos a fuerte nivel de presión, puesto que deben promover la excelencia y eficiencia en el manejo y control de servicio al cliente, además se ha denotado poca interacción entre compañeros, no existen tiempos de descanso o pausas activas, y en algunos casos se ha evidenciado trato irrespetuoso con sus compañeros, huéspedes y clientes, lo que ha afectado el ambiente de trabajo; las percepciones se enuncian como generadores de conflictos entre los trabajadores, que pueden originar baja calidad en los servicios.

En este contexto, los estilos de liderazgo, dependiendo de la integración con diferentes elementos de la organización otros aspectos organizacionales, podría ser un factor de influencia en el bienestar y el ambiente laboral, es decir, un liderazgo efectivo debe sustentarse en la productividad, y en la calidad de vida de las personas, por supuesto esto incide en el clima organizacional. Esta situación, califica al gerente, según García, et al. (2018a), desde diferentes estilos, dependiendo de su intención y capacidad de interacción, su comportamiento gerencial debe vincularse con las tomas de decisiones, el logro de los objetivos, la ejecución de las tareas y funciones complejas, que necesitan negociarse con el proceso de servicio al cliente del hotel, lo cual, pareciera estar determinada por conflictos intrapersonales, interpersonales, intergrupales o personal - funcional.

En definitiva, el capital humano es el gran activo del sector, pues este consolida los espacios de recreación, diversión y 
hospitalidad, que ofrece un destino turístico como Cartagena de Indias, por lo cual, el líder debe apoyar y propiciar su desarrollo profesional y humano dentro de la empresa. De acuerdo a lo planteado, surgió la necesidad de determinar la influencia del estilo de liderazgo en el clima organizacional del sector hotelero en Cartagena de Indias (Colombia).

\section{Liderazgo como línea de acción gerencial}

Autores como Durán y Parra (2016); así como Pacheco, et al. (2020), consideran que los factores humanos juegan un papel importante dentro de la organización, son responsables de realizar todas las tareas y/o actividades basadas en la felicidad y el progreso de la organización. Por lo tanto, es importante tener capacidades efectivas de liderazgo, orientación e integración.

En este orden de ideas, el liderazgo se entiende como la capacidad del gerente o cualquier miembro de la organización, para reorientar el comportamiento de los grupos hacia metas colectivas (Torcatt,
2020). Robbins y Coulter (2014), vinculan el liderazgo con las condiciones de trabajo, las acciones gerenciales así como a los efectos de los distintos grados de preocupación tanto de la producción como de la persona. Asi Bain (1985), asume que estos elementos, concretan la acción gerencial planteada desde la perspectiva del grupo y de la evolución natural de la organización, en procura de elevar los logros y adecuar los objetivos.

Dentro de estas perspectivas, Koontz y Weihrich (2013), creen que el liderazgo se considera el proceso de "influencia", es decir, el arte o proceso de influir en las personas para que puedan participar de forma voluntaria y entusiasta en el proceso de consecución de las metas del grupo. Asimismo, opinan que hay que animar a las personas a desarrollar la voluntad de trabajar, pero también con ilusión y confianza. Enfatizan que los líderes deben tomar medidas para ayudar al equipo a lograr sus objetivos, maximizando sus capacidades. En ese sentido, con el fin de establecer un estilo de liderazgo en el sector turístico y hotelero se determinaron los elementos que se explican a continuación en el Cuadro 1.

\section{Cuadro 1 \\ Indicadores de liderazgo}

\begin{tabular}{cl}
\hline $\begin{array}{c}\text { Elemento clave del } \\
\text { liderazgo }\end{array}$ & \multicolumn{1}{c}{ Caracterización } \\
\hline $\begin{array}{c}\text { Supervisión rigurosa } \\
\text { e interpersonal }\end{array}$ & $\begin{array}{l}\text { Robbins y Coulter (2014), manifiestan que, para que las organizaciones compitan con éxito en una economía } \\
\text { global dinámica, los empleados deben poder tomar decisiones e implementar cambios rápidamente. Por tanto, } \\
\text { creen que la implementación de la autocracia y la supervisión no participativa son los elementos que han sido } \\
\text { reemplazados en este siglo, y quienes implementan este tipo de supervisión socavan la realización de la meta. }\end{array}$ \\
$\begin{array}{c}\text { Autonomía limitada } \\
\text { por las políticas de la } \\
\text { empresa }\end{array}$ & $\begin{array}{l}\text { Para Dessler y Valera (2011), la autonomía de trabajo abarca la responsabilidad que posee cada trabajador } \\
\text { sobre su trabajo y la facilidad que tiene para realizar el mismo. La autonomía y la responsabilidad son factores } \\
\text { fundamentales a considerar dentro de un empleado, ella permite tomar decisiones propias en circunstancias } \\
\text { imprevistas, sin la necesidad de estar consultando con su superior, en tal sentido se le debe suministrar } \\
\text { herramientas de trabajo que le permitan ejercer su función principal. }\end{array}$ \\
$\begin{array}{c}\text { Métodos de } \\
\text { supervisión para }\end{array}$ & $\begin{array}{l}\text { Koontz y Weihrich (2013), consideran que, para administrar de manera efectiva, debe tener una red objetivo } \\
\text { significativa y alcanzable; a menos que las personas sepan cuál es el objetivo final de sus esfuerzos, no se } \\
\text { puede esperar que completen una tarea de manera efectiva y eficiente para producir efectividad. Por tanto, estos } \\
\text { métodos deben estar orientados a objetivos. }\end{array}$ \\
$\begin{array}{c}\text { Procesos gerenciales } \\
\text { para solucionar } \\
\text { problemas }\end{array}$ & $\begin{array}{l}\text { Robbins y Coulter (2014), sugieren que deben contar con planes estratégicos que incluyan actividades y procesos } \\
\text { que facilite la toma de decisiones, y de acciones cuando sea necesaria, esto orientado es organizaciones cuya } \\
\text { incertidumbre pueda ser elevada y los gerentes deben ser flexibles para responder a cambios inesperados. }\end{array}$ \\
\hline
\end{tabular}

Fuente: Elaboración propia, 2021. 


\section{Clima organizacional como tendencia del Liderazgo}

Brunet (2011), manifiesta que la definición de clima organizacional, se refiere a un conjunto de características que describen una organización y la distinguen de otras organizaciones, estas particularidades son relativamente estables en el tiempo y afectan el comportamiento individual dentro de ella. Además, apoyar la clasificación de elementos relacionados con el clima organizacional del departamento.
Así, Pacheco, et al. (2020) han considerado que, al analizar y realizar diagnósticos preliminares sobre el clima, las organizaciones pueden iniciar y hacer cumplir cambios que indiquen elementos específicos a los administradores que guían su intervención. De igual forma, explican los autores que el clima organizacional es relevante porque la organización lo evaluará para seguir su desarrollo y predecir cuestiones relacionadas con la percepción del entorno empresarial. A continuación, en el Cuadro 2 se evidencian los elementos del clima organizacional.

\section{Cuadro 2 \\ Elementos del clima organizacional}

\begin{tabular}{|c|c|}
\hline Elementos & Descripción \\
\hline $\begin{array}{l}\text { El ambiente permite la } \\
\text { expresión libre de ideas }\end{array}$ & $\begin{array}{l}\text { Dessler y Valera (2011) y Brunet (2011), se refieren al deseo de los empleados y } \\
\text { gerentes de brindar sus opiniones para ayudar a resolver problemas organizacionales } \\
\text { y tomar conciencia de los riesgos. Este elemento del clima organizacional según } \\
\text { lo indicado por Dessler y Valera (2011), incluye simpatía informal y grupos de } \\
\text { trabajo, trato considerado al personal de la empresa, tratamiento de los errores } \\
\text { como una forma de apoyo y aprendizaje y, finalmente, recompensa a los empleados } \\
\text { de manera justa por su trabajo. }\end{array}$ \\
\hline $\begin{array}{c}\text { Programas para incrementar } \\
\text { la eficiencia }\end{array}$ & $\begin{array}{l}\text { Dessler y Valera (2011) y Martínez (2016), asumen que facilita la adaptación } \\
\text { al puesto de trabajo, contribuye a analizar las características del puesto } \\
\text { desempeñado, así como su entorno, revisión de los objetivos previstos en cada } \\
\text { puesto de trabajo y detectar la capacitación tanto personal como colectivo. }\end{array}$ \\
\hline Trabajo bajo presión & $\begin{array}{l}\text { Según Herriegel y Slocum (2009), se generan procesos de mayor exigencia } \\
\text { personal, promoviendo la participación de ellos en múltiples actividades formales } \\
\text { e informales en las empresas para las cuales trabaja, además de que se produce un } \\
\text { alto nivel de exigencia en el desarrollo de las actividades para lograr cumplir con } \\
\text { las metas trazadas. }\end{array}$ \\
\hline $\begin{array}{c}\text { Recompensas por los logros } \\
\text { alcanzados }\end{array}$ & $\begin{array}{l}\text { Para Brunet (2011), corresponde a la percepción que tiene el miembro de } \\
\text { las recompensas por el trabajo sobresaliente. Este es el grado en el que la } \\
\text { organización usa recompensas en lugar de castigos. Esta dimensión puede crear } \\
\text { un ambiente apropiado en la organización, pero mientras no sean castigados, } \\
\text { alientan a los empleados a hacer bien su trabajo, y si no lo hacen bien, recuerdan } \\
\text { Mejora a medio plazo. }\end{array}$ \\
\hline
\end{tabular}

Fuente: Elaboración propia, 2021.

\section{Metodología}

Esta investigación se clasifica como descriptiva y relevante, porque intenta describir las variables de estilo de liderazgo y clima organizacional en un momento dado. Asimismo, su diseño es no experimental, puesto que el estudio no involucró una manipulación deliberada de variables, las cuales son tomadas directamente de la industria hotelera en Cartagena de India (Colombia), especialmente de la situación actual que se genera en el sector Bocagrande. También, es transaccional porque los datos se recopilan en un solo momento, lo 
que brinda una oportunidad para el desarrollo de aplicaciones de instrumentos. Además, se dice que la investigación se realizó en sitio, porque los datos fueron recolectados directamente en el lugar de contacto con los sujetos del componente poblacional o del sitio de investigación.

La población estuvo conformada por 35 empleados operativos de 5 hoteles ubicados en el sector Bocagrande de Cartagena de Indias, esta población es finita por estar constituida por menos de cien mil unidades y es accesible, denominado censo poblacional. Para recolectar los datos se utilizaron 2 instrumentos tipo cuestionario con preguntas cerradas, con cinco (5) posibilidades de respuestas tipo Likert. A este instrumento se le aplicó la validez de contenido, a través del juicio de 5 expertos, posteriormente, se procedió a determinar la confiabilidad a través del método Alfa Crombach, el cual dio como resultado un coeficiente 0,84 .

El análisis de los datos, se realizó a través de la estadística descriptiva, los mismos fueron procesados a partir de un baremo de interpretación, el cual se construyó sobre la base de las puntuaciones utilizadas en la escala, la cual va de 1 a 5 . Ubicándose en los rangos muy bajos $(1-1,79)$, bajo $(1,80-2,59)$, medio $(2,60-3,39)$, alta $(3,40-4,19)$ y muy alta $(4,20-5)$. Posteriormente, se procedió a calcular el índice de correlación, estimándolo con el índice de Pearson y verificar en qué medida el estilo de liderazgo influye en el clima organizacional. Este coeficiente de correlación de Pearson, es un índice cuyos valores absolutos oscilan entre 0 y 1 . Y se seleccionó un nivel de significancia de $\alpha=0,05$.

\section{Resultados y discusión}

Con respecto al estilo de liderazgo, en la Tabla 1 se evidencia una marcada tendencia a la poca aplicación de herramientas estratégicas para estructurar las operaciones en función de unificar los equipos de trabajo, promoviendo una supervisión rigurosa e interpersonal, dado que el promedio se ubicó en la categoría medio con un rango de 3 , y la desviación estándar se ubicó en 1,382; destacando una dispersión de rango medio respecto al indicador analizado, generando como consecuencia que las actividades se vean afectadas por las presiones ejercidas. A este respecto, Parra, et al. (2018) mencionaron que algunas empresas dan importancia a la producción, pero no consideraron la retroalimentación de la comunicación y la distancia entre el subordinado y el superior.

\section{Tabla 1}

Estilo de liderazgo

\begin{tabular}{lcccc}
\multicolumn{1}{c}{ Indicador } & Media & Moda & Mediana & DS \\
\hline Supervisión rigurosa e interpersonal & 3,0 & 3,0 & 3,0 & 1,382 \\
Procesos gerenciales, para solucionar problemas & 3,2 & 4,0 & 3,0 & 1,437 \\
Autonomía limitada por las políticas de la empresa & 2.6 & 2,0 & 3,0 & 1,248 \\
Métodos de supervisión, para fomentar la eficacia & 2.4 & 1.0 & 2 & 1.20 \\
\hline
\end{tabular}

Fuente: Elaboración propia, 2021.

Con relación a este proceso, se evidencia lo planteado por Pacheco, et al. (2020), en su estudio de clima organizacional tomando como referencia, si la estructura de las operaciones laborales involucra procesos gerenciales para solucionar los problemas laborales generados en la organización y con el cliente, y se trasladó al área operativa de los hoteles, en la cual existe una clara tendencia hacia una acción de categoría media, con un rango en el promedio de 3.2 , sustentado con una desviación estándar de 1.437. 
Asimismo, se pudo constatar la existencia de aptitudes hostiles, en este sentido, se evidenció que los trabajadores consideraban tener algunas diferencias en cuanto al clima que se percibe, asi como se interactúa en el espacio laboral. Destacando, que el clima que prevalece o se descubre, realmente depende es del conjunto de actores que hacen vida, y no solo del empleado existente, es decir, no está en manos del empleado, sino de los efectos del entorno organizacional.

A este respecto, Dessler y Valera (2011); y García, et al. (2018b), establecen que las estructuras formales de las organizaciones deben estar alineadas en cuanto a sus políticas y responsabilidades, así como la atmósfera requerida, lo cual influye en la planeación adecuada dentro de la organización para lograr los objetivos propuestos.

Posteriormente, la Tabla 1 refleja los datos logrados cuando se le preguntó al personal si la autonomía en su puesto depende de las políticas del área gerencial del hotel, demostrando que se ubica en una categoría baja y el límite mínimo de la categoría media, puesto que su rango tiene un promedio de 2,6 así como una desviación estándar de 1,248; incurriendo en que la autonomía en los puestos de trabajo, está limitada por las políticas de la institución, y que para ejecutar alguna actividad fuera de sus funciones esta debe ser consultada y esperar la aprobación.

En este sentido, Parra, et al. (2018) asumieron que la autonomía de trabajo abarca la responsabilidad que posee cada trabajador sobre su trabajo y la facilidad que tiene para realizar el mismo. Por otra parte, García, Durán y Prieto (2017), manifiestan que la autonomía y la responsabilidad, son factores fundamentales a considerar dentro de un funcionario de la empresa, puesto que ellas le permiten tomar decisiones propias en circunstancias imprevistas, sin la necesidad de estar consultando con su superior, en tal sentido, se le debe suministrar herramientas de trabajo que le consientan ejercer su función principal.
De acuerdo con lo planteado, el estilo de liderazgo en este contexto se relaciona con la colaboración del supervisor con sus empleados y su compañeros, lo cual en opinión de García, et al. (2017b), se hace en pro de ser más competitivo, la fijación de objetivos, orientación y seguimiento de las acciones, del personal a su cargo, para liderar al equipo de trabajo; en este ámbito, se manifiesta un promedio de 2,4 (ver Tabla 1) ubicándose en una categoría baja, de acuerdo al promedio de interpretación de la variable, destacando que el estilo de liderazgo orientado al proceso y participativo que requiere el sector turístico no es el que prevalece, pues se genera tendencia hacia un liderazgo autocrático, de acuerdo con lo manifestado por Newstron (2015).

Aunado a ello, se toma en cuenta la opción que proponen Benavides y Quintana (2003), así como García, et al. (2017b); señalando que el liderazgo es la capacidad de desempeñar el papel de un equipo o líder de equipo. Además, las capacidades de gestión representan un conjunto de atributos personales obvios, que contribuyen al trabajo y se consideran comportamientos para lograr un desempeño efectivo. Lo que no está sucediendo en la industria hotelera hoy. Por otro lado, Hollloway (2012); y Pineda (2020), sostienen que el estilo del líder, debe expresar la cultura y los valores de la organización para maximizar a sus empleados y hacer lo mejor en cada tarea.

En la Tabla 2, se muestran los indicadores relacionados con las características del clima organizacional predominante en los hoteles seleccionados. En lo que concierne al ambiente permisible para expresar libremente las ideas, se observa un promedio de 2,1 ubicándose en una tendencia de categoría baja respecto al baremo de interpretación, aunado a una desviación estándar de 0,90; en este sentido, el ambiente no permite la expresión libre de las ideas, lo que puede obstaculizar posibilidades de líneas de acción a ejecutar y promover una atención asertiva al cliente. 
Tabla 2

Características del de clima

\begin{tabular}{lcccc}
\hline \multicolumn{1}{c}{ Indicador } & Media & Moda & Mediana & DS \\
\hline El ambiente permite la expresión libre de ideas & 2.1 & 2,00 & 2.00 & 0.90 \\
Programas para incrementar la eficiencia & 2,40 & 1,00 & 2,00 & 1,20 \\
Trabajo bajo presión & 3,60 & 4,00 & 4,00 & 1,30 \\
Recompensas por los logros alcanzados & 2,60 & 2,00 & 2,00 & 1.1 \\
\hline
\end{tabular}

Fuente: Elaboración propia, 2021.

En este caso, Dessler y Valera (2011) y Brunet (2011), hacen referencia al deseo de los empleados de ofrecer sus opiniones que ayuden a resolver los problemas de la organización y al sentido de correr riesgos. Si el sector hotelero tomase en cuenta las opiniones de los empleados, tendría posibilidad de resolver situaciones con mayor celeridad y ofrecer un servicio óptimo al cliente.

Con respecto a la existencia de programas de orientación para incrementar la eficiencia del personal operativo, se alcanzó un promedio de 2,40 ubicándolo en una categoría baja respecto a su existencia, con una desviación estándar de 1,20 (ver Tabla 2); lo que indica, que no existen programas de orientación para el personal que incremente su eficiencia en los procesos y tareas ejecutadas. Dentro de estas perspectivas, Durán y Parra (2016), asumieron que las organizaciones modernas deben orientarse a brindar al recurso humano unas condiciones favorables y un ambiente agradable de trabajo, de esta manera podrían alcanzar sus objetivos; para ello, deben adaptarse a los procesos de transformación y cambios concebidos desde el entorno, tal y como se debe realizar en el sector hotelero en Cartagena de Indias.

En cuanto a la presión en el trabajo, la media aritmética destacada en la Tabla 2, indica que existe un alto nivel de presión con un rango de 3,60, lo que indica que en el sector hotelero las acciones laborales están sometidas a altas presiones, incidiendo de forma directa en situaciones personales y por ende en el clima organizacional. Brunet (2011), considera que, al existir presión en el trabajo, el trabajador podría adoptar una actitud negativa debido al clima organizacional percibido, por lo cual, desarrollará una actitud aún más afanosa cuando vea los resultados de la organización y mucho más si la productividad baja.

De acuerdo a las recompensas que reciben por los logros alcanzados, se observa en la Tabla 2, un promedio de 2,60, generando una categoría de nivel mínimo medio con tendencia a baja. Con una dispersión de 1,1 en su desviación estándar. En consecuencia, en los hoteles que forman parte de la población del estudio, las recompensas otorgadas hacia los logros no son equivalentes al esfuerzo realizado por el personal.

García, et al. (2018b), reconocen la importancia de algunas estrategias de gestión para permitir la implementación y ejecución de medidas transformadoras e innovadoras que puedan asegurar el proceso, pero al mismo tiempo, asegurar que la organización tenga la capacidad de orientar a los subordinados para completar las tareas asignadas. Deben valorar plenamente la cooperación de sus miembros, establecer un mecanismo para que tengan la motivación suficiente para mejorar la eficiencia y el desempeño, logrando así las metas organizacionales.

Para dar respuesta a la investigación orientada a determinar la relación entre estilo de liderazgo y clima organizacional en el sector hotelero en Cartagena de Indias, se aplicó el Coeficiente de Correlación de Pearson, y los cálculos fueron realizados en el programa SPSS 22.0, asignándoles la siguiente nomenclatura para cada una de las variables: $\mathrm{X}=$ Estilos de liderazgo $\mathrm{Y}=\mathrm{Clima}$ organizacional. En este sentido, los resultados obtenidos se muestran en la siguiente Tabla 3. 
Tabla 3

Cálculos de correlación de Pearson

\begin{tabular}{ccccc}
\hline $\mathbf{X}$ & $\mathbf{Y}$ & $\mathbf{X} * \mathbf{X}$ & $\mathbf{Y} * \mathbf{Y}$ & $\mathbf{X} * \mathbf{Y}$ \\
\hline 3901,00 & 5011,00 & 441981 & 728293 & 566166 \\
& & & 0,866371956 & \\
\multicolumn{2}{l}{ Coeficiente de Correlación } & & \\
\hline
\end{tabular}

Fuente: Elaboración propia, 2021.

Al realizar los cálculos del coeficiente de Pearson, los resultados arrojaron una correlación de 0,866 , lo cual muestra que la correlación entre las variables de estudio es positiva muy alta; es decir, que el estilo de liderazgo aplicado en el sector incide de una manera significativa en el clima organizacional que se ha generado en los hoteles que forman parte de la población en estudio.

Se cita a Durán, Parra y García (2018), así como Pacheco, et al. (2020), quienes consideran que las competencias directivas como comportamientos observables y habituales pueden posibilitar el éxito de una persona en su función operativa. En este sentido, Pérez (1996); así como Vélez, et al. (2019), evidencian que la función directiva se enfoca en diseñar estrategias que produzcan valor económico, desarrollando las capacidades de sus empleados, convirtiéndolos en profesionales integrales, y uniéndolos con la misión de la empresa, lo cual producirá un clima organizacional favorable, que permita fomentar en los equipos, valores como la responsabilidad, disciplina, generación de ideas, que conlleven al logro de las metas.

\section{Conclusiones}

Las conclusiones permiten inferir que el estilo de liderazgo en el sector hotelero está orientado a un proceso autocrático, el líder despliega comportamientos que no hacen una combinación hacia las tareas con las personas, por lo cual, no genera eficacia en las acciones, no contribuye al compromiso, participación y satisfacción, su tendencia es orden, soluciones y consistencia. Por tanto, el funcionamiento de las organizaciones investigadas se relaciona con la actitud de los colaboradores, su comportamiento y la forma en que se ejecuta el proceso. En este sentido, el clima está determinado por aspectos como el comportamiento, los talentos, las expectativas de los demás y las realidades sociales así como culturales del departamento. Estas características forman parte del comportamiento y la integración de los empleados.

Dentro de este marco, los hechos han demostrado que no hay autonomía en el trabajo, porque los empleados no adoptan las líneas normativas para el desempeño del mismo, debido a que realizan métodos de trabajo alineados a las tendencias de la organización. También muestran los lideres un método de supervisión inflexible e impreciso. Por otra parte, el entorno laboral es rígido, lo que les trae grandes riesgos.

En cuanto al posicionamiento de la empresa, no existe un plan de desarrollo laboral, lo que significa una tendencia de poca autoeficacia en el empleado. En este sentido, los investigadores consideran que, para lograr la eficacia en las organizaciones del sector hotelero de Cartagena de Indias, se deben emplear los métodos bajo una orientación de servicio práctico, acentuando y destacando la importancia de hacer una normativa interna en cuanto a sus operaciones $\mathrm{y}$ acciones, incluido los recursos, por supuesto que esto conlleva una gestion basada en la conservación y control como sujetos que son parte de un conglomerado social o grupo social organizado, promoviendo procesos de 
bienestar y un clima organizacional favorable al servicio que el cliente espera.

Ello debe tener implicaciones profundas, generando relaciones evidentes con la productividad, la comunicación, toma de decisiones, la eficiencia, el éxito y sobre todo en el bienestar de su gente, por cuanto condiciona el comportamiento de las personas en la organización.

Finalmente, se denota que las organizaciones del sector hotelero requieren de liderazgos fuertes y administración sólida para obtener una eficacia óptima, por lo cual, se necesitan líderes que desafíen el statu quo, para crear visiones del futuro e inspirar a los miembros de la organización, con la finalidad que deseen lograr las visiones y ser competitivos, manteniendo una imagen de calidad de servicio al cliente del mejor nivel. También, se necesitan gerentes que formulen planes detallados, creen estructuras organizacionales eficientes y supervisen las operaciones cotidianas, pero indudablemente, promoviendo el bienestar del empleado y consolidando un clima organizacional eficiente.

\section{Referencias bibliográficas}

Bain, D. (1985). Productividad, la solución a los problemas de la empresa. McGraw-Hill.

Benavides, C. A., y Quintana, C. (2003). Gestión del conocimiento y calidad total. Diaz de Santos.

Brunet, L. (2011). El clima de trabajo en las organizaciones. Editorial Trillas.

Chiavenato, I. (2013). Comportamiento organizacional. La dinámica del éxito en las organizaciones. Editorial McGraw-Hill.

Dessler, G., y Valera, R. (2011). Administración de recursos humanos. Un enfoque latinoamericano. Pearson Educación.
Durán, S. E., y Parra, M. A. (2016). Condiciones laborales y calidad de vida en el trabajo: Un desafío para la gestión de talento humano en Venezuela. En R. Prieto y R. De La Hoz (Eds.), Estrategias innovadoras: Afrontando los retos de la economía global (pp. 437-459). Universidad Autónoma del Estado de Hidalgo y Universidad Autónoma de Tlaxcala.

Durán, S. E., Parra, M. A., y García, J. (2018). El aprendizaje como técnica de mejora continua en la gestión de desarrollo organizacional en Pymes Colombianas. Ediciones Adolfo Pontigo Loyola.

García, J. E., Durán, S. E., y Prieto, R. (2017). Políticas de gestión de talento humano para el desarrollo de competencias gerenciales en empresas metalmecánica. FACE: Revista de la Facultad de Ciencias Económicas $y$ Empresariales, 17(2), 130-141. https://doi.org/10.24054/01204211. v2.n2.2017.2657

García, J. E., Durán, S. E., Parra, M. A., y Márceles, V. S. (2017a). Dirección estratégica del talento humano para el fomento de valores en los cuerpos policiales venezolanos. Revista Espacios, 38(32), 16.

García, J. E., Durán, S. E., Cardeño, E., Prieto, R., García, E., y Paz, A. (2017b). Proceso de planificación estratégica: Etapas ejecutadas en pequeñas $\mathrm{y}$ medianas empresas para optimizar la competitividad. Revista Espacios, 38(52), 1-14.

García, J. E., Durán, S. E., Hernández, J. C., y Moreno, M. E. (2018a). Estrategias gerenciales para fomentar las competencias laborales en el sector hotelero de la costa caribe colombiana. Revista de Investigación SIGMA, 5(2), 8-22. https://dx.doi.org/10.24133/ sigma.v5i02.1251 
García, J. E., Durán, S. E., Parra, M., y García, E. (2018b). Elementos y principios de la calidad de servicio en instituciones de educación superior. En R. A. Prieto y R. J. De La Hoz (Eds.), Marketing y competitividad en las organizaciones: Enfoque y perspectivas (pp. 327-353). Universidad Autónoma del Estado de Hidalgo y Universidad Autónoma de Tlaxcala.

Herriegel, D., y Slocum, J. W. (2009). Comportamiento organizacional. Cengage Learning Editores.

Holloway, J. B. (2012). Leadership behavior and organizational climate: An empirical study in a non-profit organization. Emerging Leadership Journeys, 5(1), 9-35.

Koontz, H., y Weihrich, H. (2013). Elementos de administración: Un enfoque internacional y de innovación. McGraw-Hill/ Interamericana Editores.

Martínez, J. (2016). Inversión para la formación del talento humano en empresas farmacéuticas del estado Zulia - Venezuela. Revista Venezolana de Gerencia, 21(76), 642-652.

Martínez-Ventura, J., Cardeño-Portela, E., Ramírez-Cardeño, W., y Durán, S. (2017). Liderazgo transformacional como estrategia de adaptación en la gestión logística empresarial. Desarrollo Gerencial, 9(2), 140$157 . \quad$ https://doi.org/10.17081/ dege.9.2.2980

Newstron, J. W. (2015). Comportamiento humano en el trabajo. Mc Graw Hill/ Interamericana Editores.

Pacheco, C. M., Rojas, C. P., Niebles, W. A., Hernández, H. G., y Durán, S. E. (2020). Estrategias motivacionales para caracterizar el clima organizacional en el sector salud. Revista Espacios, 41(29), 1-17.
Parra, M., Durán, S. E., Márceles, V., Yarzagaray, J., Valenzuela, I., Payares, K., Alvarino, C., Navarro, N., Charris, P., y Ramírez, J. (2018). Factores del clima organizacional existentes en el área administrativa en empresas de Barranquilla. Revista Espacios, 39(50), 21-35.

Parra, M., Visbal, O., Durán, S. y Badde, G. (2019). Calidad de la comunicación y actitud de los empleados ante procesos de cambio organizacional. Interdisciplinaria Centro Interamericano de Investigaciones Psicológicas y Ciencias Afines, 36(1), 155-170. https://doi.org/10.16888/ interd.2019.36.1.11

Pérez, J. A. (1996). Fundamentos de la dirección de empresas. Ediciones Rialp.

Pineda, L. (2020). Aproximación teórica al concepto de calidad y los sistemas de gestión. SUMMA. Revista Disciplinaria en Ciencias Económicas y Sociales, 2(1), 41-62.

Robbins, S. P., y Judge, T. A. (2017). Comportamiento organizacional. Editorial Pearson Education.

Robbins, S. P., y Coulter, M. (2014). Administración. Editorial Pearson Education.

Torcatt, T. J. (2020). Liderazgo gerencial y desempeño laboral en docentes del estado Nueva Esparta, Venezuela. Revista de Ciencias Sociales (Ve), $X X V I(\mathrm{E}-2), \quad 42-53 . \quad \mathrm{https}: / / \mathrm{doi}$. org $/ 10.31876 /$ rcs.v26i0.34112

Vélez, O. I., Beltrán, J. A., López, J. A., y Arias, F. J. (2019). Asociatividad empresarial y liderazgo ambidiestro como generadores de innovación. Revista de Ciencias Sociales (Ve), $X X V(2), 51-72$. 